training scheme. When asked if a trainee encountered significant challenges in accessing training - CT/MRI, followed by Adult Congenital Heart Disease and Electrophysiology were most frequently identified. Interest in dual accreditation was low, with $77 \%$ of respondents saying they did not intend to purse dual accreditation. Approximately half (47\%) of respondents felt that their year spent doing General Internal Medicine (GIM) enhanced their training experience. 46\% of respondents felt they have a poor or very poor work-life-balance, but $72 \%$ indicated that they had a very good or good quality of life. Future career planning: $83 \%$ of trainees indicated definitely or probably when asked if planning to work in Ireland after completion of training. However, when in 2018, a small number of trainees on overseas out of program experience were asked the same question a smaller proportion of respondents indicated that they wished to work in Ireland. In relation to gender - $28 \%$ of respondents were female, in keeping with prior work published by the authors in 2017 showing a 7:3 gender ratio overall for trainees between 1998 and 2017. To address the gender balance respondents felt that changes to the training scheme(63\%) and work practices (72\%) would be needed. Covid-19 had a marked affect on training, with $94 \%$ of trainees responding that it adversely affected their training.

Conclusion This 4 year study of the Irish cardiology trainees gives important insights into the strengths and weaknesses of the training scheme. It highlights the impact due to the current Covid-19 pandemic on training. It also gives valuable information regarding the future career goals of current trainees. All of the above, should be useful in the framing and shaping of any discussion regarding the future of cardiology training and service delivery in Ireland in the decades to come.

\section{BENEFITS OF 3D PRINTING IN PLANNING AND COMMUNICATION IN ADULT CARDIAC SURGERY: A PILOT STUDY}

${ }^{1} \mathrm{G}$ Beattie, ${ }^{2} \mathrm{~A}$ Graham. ${ }^{1}$ Royal Victoria Hospital, Belfast, UK; ${ }^{2}$ Belfast Trust, Belfast UK

\subsection{6/heartjn|-2021-ICS.20}

Introduction 3D printing is now cost effective and, with specialist companies handling the complexity of making models, it is now relatively easy to get an accurate surgical model printed. Simulation surgery is now becoming a reality to highlight areas of concern in an operation. Some orthopaedic surgeons are using models to practice the operation before ever laying a hand on a patient, so the authors wanted to investigate if $3 \mathrm{D}$ printed models would help with planning and communication in adult cardiac surgery.

Methods A local company who prints 3D medical models was given a stimulus grant to from June 2020 to March 2021. They were able to offer models for free to local specialist surgery departments. When the surgery was complete an online assessment form was completed to review the helpfulness of the models, and all of these forms were collated from the company for the cardiac models ordered during the study period. The data was analysed in Microsoft Excel. CT scans were sent electronically to the company. They created the model using proprietary software and an interactive model was sent to the surgeon using sketchfab. This was viewed by the requesting surgeon who made changes as necessary. When

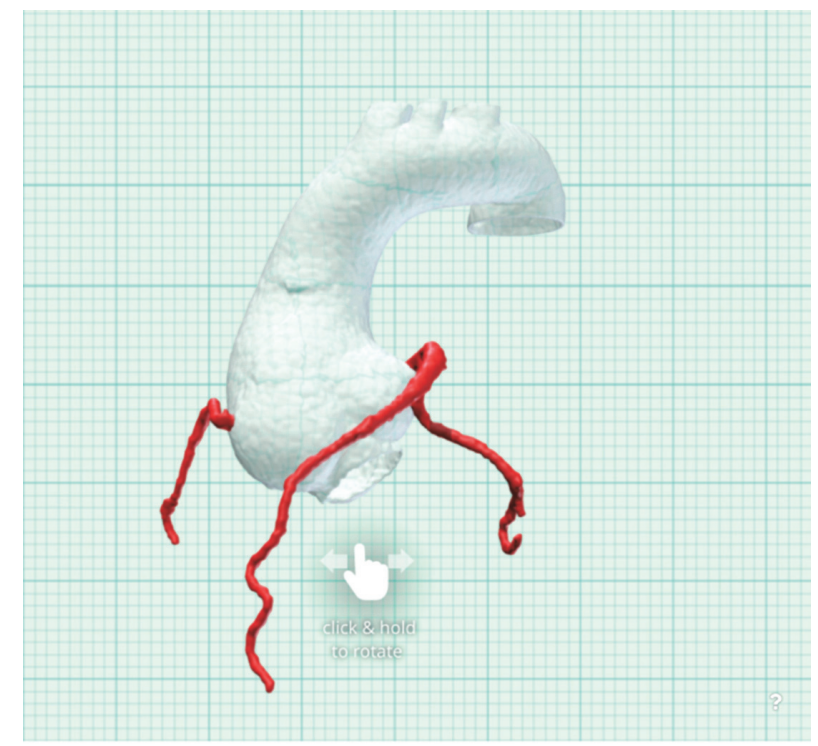

Abstract 20 Figure 1 Image of the model as it appears to the surgeon. In the software it is interactive so the surgeon can rotate and if the model is sliced this can be manipulated to allow viewing of the internal structure of the heart model

Link to interactive image: https://sketchfab.com/3d-models/p03018db0590ae83954351877edcc38776e6a2

the model was approved it was then printed. Different materials could be requested differing in flexibility to opacity. The company used Formlabs 2 and Formlabs 3B printers.

Results Sixteen 3D Models were made to aid planning of adult cardiac surgery, 6 for valve surgery, 4 for aortic root surgery, 4 for CABG surgery, 1 for a myxoma and 1 case for post-infarction VSD. In 9 cases (56\%), the surgeon changed their plan for the surgery after viewing the model and in 2 cases $(12 \%)$ it changed the diagnosis. In 15 cases (94\%) the model was felt to have a positive effect on patient safety and in all cases it was felt to be helpful for communication with other surgeons and trainees.

Conclusion Our pilot study suggests that 3D printed models have the potential to improve safety and communication in certain types of adult cardiac surgery and merit further study. Link to interactive image https://sketchfab.com/3d-models/ p03018-db0590ae83954351877edcc38776e6a2.

\section{SURGICAL AORTIC VALVE REPLACEMENT: EARLIER VS NEWER GENERATION BIOPROSTHETIC VALVES - A COMPARISON OF EARLY HEMODYNAMIC PERFORMANCE}

${ }^{1,2} \mathrm{G}$ Keehan, ${ }^{1} \mathrm{~A}$ Vainorius, ${ }^{1} \mathrm{~A}$ White, ${ }^{1} \mathrm{~A}$ Soo. ${ }^{1}$ Department of Cardiothoracics, University Hospital Galway, Ireland; ${ }^{2}$ National University of Ireland, Galway

\subsection{6/heartjnl-2021-ICS.21}

Objectives Modern advances in bioprosthetic valve tissue technology have resulted in the development of newer generation bioprosthetic aortic valves such as the Edwards Lifesciences ${ }^{\circledR}$ Inspiris Resilia ${ }^{\mathrm{TM}}$ (Inspiris) and the Medtronic ${ }^{\circledR}$ Avalus $^{\mathrm{TM}}$ Bioprosthesis (Avalus).

These claim to exhibit improved hemodynamic sustainability, and prevention from structural valve deterioration, translating into long-term durability; in comparison to bioprosthetic 
valves previously used in surgical aortic valve replacement. While long term data on these newer generation valves does not yet exist to compare durability between these two groups, it is possible to compare hemodynamic profiles with the use of doppler echocardiography post-operatively.

Our study sought to evaluate the effect of aortic valve type implanted on both max peak gradient (maxPG), and mean peak gradient (meanPG) across the aortic valve post-operatively, taking account of valve size used intraoperatively; through the use of a prospective, non-randomized, interventional study.

Methods Patients who received a biologic aortic valve replacement between July 2017 and May 2021 in our centre were studied by echocardiography in the early post-operative period. Patients who received a sutureless aortic valve, and those who died prior to post-operative echocardiogram were excluded. The remaining population comprised 106 patients (Avalus $=23$, Inspiris $=9$ ). Other valves $(n=74)$ including Edwards Lifesciences Perimount Magna Ease $(n=14)$, LinaNova Aortic pericardial heart valve $(n=22)$, Medtronic Mosaic Bioprosthesis $(n=22)$, Medtronic Mosaic Ultra Bioprosthesis $(n=6)$, St Jude Trifecta $(n=9)$ and St Jude Epic bioprosthesis $(n=1)$ were grouped and analysed collectively as older generation aortic valves.

Results Edwards Lifesciences ${ }^{\circledR}$ Inspiris ResiliaTM were associated with statistically significant lower meanPGs in comparison to the meanPGs seen after implantation of Medtronic ${ }^{\circledR}$ Avalus ${ }^{\mathrm{TM}}$ Bioprosthesis of the same size $(\mathrm{p}=0.001)$. In addition to this, despite the small sample size analysed, a statistically significant difference was seen in post-operative meanPGs between the Inspiris and the previous generation bioprosthetic aortic valves $(p=0.007)$, taking the contributory effect of valve size on pressure gradient into account. In contrast, there appeared to be no statistically significant difference in hemodynamics post Avalus in comparison to the older generation bioprosthetic aortic valves.

Conclusions Limitations of this study included the potential effect of other confounding factors such as ejection fraction and patient's body surface area on post-operative doppler gradients, that were not accounted for in this study. Despite this, and the small sample size analysed, the results seen from Inspiris bioprosthesis appear promising in this small pilot study; showing favourable flow-gradient patterns at time of early post-operative transthoracic echocardiogram, in comparison to the flow-gradient patterns seen post-operatively following implantation of previous generation aortic valves.

\section{UTILISATION AND YIELD OF GENETIC TESTING AT AN INHERITED CARDIAC CONDITIONS CLINIC}

C Galvin, M Gallagher, H Cronin, C McGorrian, T Prendiville, J Galvin. Mater Misericordiae University Hospital/University College Dublin/Family Heart Screening Clinic, Dublin, Ireland

\subsection{6/heartjnl-2021-ICS.22}

Background This study was undertaken to compare the proportions of conditions referred/detected in probands of at-risk families at a single Inherited Cardiac Conditions (ICC) clinic and to assess the uptake of genetic testing and the yield of actionable genetic testing results in these individuals.

Methods In this retrospective study, we interrogated the clinic's proband database $(n=1,817$; representing a total of 3,316 condition-affected persons) in July 2020. We extracted data about those whose primary diagnosis was one of six ICCs: Hypertrophic Cardiomyopathy (HCM; n=444), Dilated Cardiomyopathy (DCM; $n=235)$, Long QT Syndrome (LQTS; $\mathrm{n}=220$ ), Arrhythmogenic Cardiomyopathy (ACM; n=30), Brugada Syndrome $(\mathrm{BrS} ; \mathrm{n}=25)$ and Catecholaminergic Polymorphic Ventricular Tachycardia (CPVT; $n=9$ ). We also included Sudden Adult Death Syndrome (SADS; $\mathrm{n}=165$ ) as an additional category. Genetic variants which were classified as Pathogenic (P), Likely Pathogenic (LP) and Variant of Uncertain Significance (VUS) by American College of Medical Genetics were grouped together as 'not negative'. $P$ and LP variants are directly clinically actionable while VUSs are not.

Conclusion The overall utilisation of genetic testing was relatively low at $32.4 \%$ in this cohort. The overall not negative (P, LP or VUS) rate for genetic testing across the ICCs probands in whom genetic testing was pursued was $60.7 \%$. Combined $\mathrm{P} / \mathrm{LP}$ variant rates within the not negative group in the larger cohorts of HCM $(n=86)$ and LQTS $(n=88)$ were $82.5 \%$ and $84.7 \%$ respectively. VUS rates vary from $0 \%$ to

\begin{tabular}{|c|c|c|c|c|c|c|c|c|c|}
\hline \multicolumn{10}{|c|}{ Abstract 22: Results } \\
\hline Condition & $n=$ & $\begin{array}{l}\text { No } \\
\text { Genetics }\end{array}$ & Genetics & $\begin{array}{l}\text { Genetics - } \\
\text { ve }\end{array}$ & $\begin{array}{l}\text { Genetics not - } \\
\text { ve }\end{array}$ & Pathogenic* & $\begin{array}{l}\text { Likely } \\
\text { Path* }\end{array}$ & VUS* & $\begin{array}{l}>1 \\
\text { variant }\end{array}$ \\
\hline HCM & 444 & $\begin{array}{l}290 \\
(65.3 \%)\end{array}$ & $\begin{array}{l}154 \\
(34.7 \%)\end{array}$ & $68(44.2 \%)$ & $86(55.8 \%)$ & $58(67.4 \%)$ & $13(15.1 \%)$ & $\begin{array}{l}14 \\
(16.3 \%)\end{array}$ & 10 \\
\hline DCM & 235 & $\begin{array}{l}193 \\
(82.1 \%)\end{array}$ & $42(17.9 \%)$ & $15(35.7 \%)$ & $27(64.3 \%)$ & $9(33.3 \%)$ & $11(40.7 \%)$ & $7(25.9 \%)$ & 5 \\
\hline LQTS & 220 & $98(44.5 \%)$ & $\begin{array}{l}122 \\
(55.5 \%)\end{array}$ & $37(30.3 \%)$ & $85(69.7 \%)$ & $51(60 \%)$ & $21(24.7 \%)$ & $\begin{array}{l}13 \\
(15.3 \%)\end{array}$ & 9 \\
\hline ACM & 30 & $17(56.7 \%)$ & $13(43.3 \%)$ & $3(23 \%)$ & $10(77 \%)$ & $6(60 \%)$ & $1(10 \%)$ & $3(30 \%)$ & 1 \\
\hline $\mathrm{BrS}$ & 25 & $18(72 \%)$ & $7(28 \%)$ & $2(28.6 \%)$ & $5(72.4 \%)$ & $2(40 \%)$ & $3(60 \%)$ & $0(0 \%)$ & 0 \\
\hline CPVT & 9 & $6(66.7 \%)$ & $3(33.3 \%)$ & $1(33.3 \%)$ & $2(66.7 \%)$ & $2(100 \%)$ & $0(0 \%)$ & $0(0 \%)$ & 0 \\
\hline SADS & 165 & $\begin{array}{l}140 \\
(84.8 \%)\end{array}$ & $25(15.2 \%)$ & $18(72 \% \%)$ & $7(28 \%) \wedge$ & $0(0 \%)$ & $0(0 \%)$ & $7(100 \%)$ & 0 \\
\hline Total & 1128 & $762(67.6)$ & $\begin{array}{l}366 \\
(32.4 \%)\end{array}$ & $\begin{array}{l}144 \\
(39.3 \%)\end{array}$ & $222(60.7 \%)$ & $128(57.7 \%)$ & $49(22.1 \%)$ & $\begin{array}{l}44 \\
(19.8 \%)\end{array}$ & 25 \\
\hline
\end{tabular}

'Genetics -ve' and 'Genetics not -ve'\%s calculated from the 'Genetics' totals. 'Pathogenic', 'Likely Pathogenic' or 'VuS'\%s calculated from the 'Genetics not -ve' totals. *Where multiple variants were detected in a single individual, their most pathogenic variant was recorded in this table. SADS patients with a P or LP variant were moved to the appropriate other condition. 